\title{
Gate keeping and legitimisation were central in the interactions between informal carers of older people and healthcare workers
}

\author{
May J, Ellis-Hill C, Payne S. Gatekeeping and legitimization: how informal carers'relationship with health care workers is \\ revealed in their everyday interactions. J Adv Nurs 2001 Nov;36:364-75.

\section{QUESTION: What processes are involved in everyday face to face interactions between informal carers of older people and healthcare workers in a hospital setting?}

\section{Design}

Ethnomethodology.

\section{Setting}

A rehabilitation ward for older people in a community based hospital in the south of England, UK.

\section{Participants}

19 English speaking carers who attended the ward and 25 healthcare workers. Informal carers were those who provided physical or social care or assistance for another person. Without this help, the recipient would need health, social, or voluntary sector service intervention. Healthcare workers were nurses, physiotherapists, occupational therapists, dieticians, ambulance personnel, nursing auxiliaries, agency carers, and housekeepers.

\section{Methods}

A static videotape recorder continuously monitored activity at and around the ward desk. The social interactions and speech of informal carers and healthcare workers were recorded. 1-3 hour recordings were done 2-3 times a week over a period of 3 months (30 recorded interactions). Videotape transcripts recorded the actions and movements of the participants as well as their verbal and paralinguistic behaviours (eg, simultaneous utterances, silences, pauses, and intonation). The transcripts were analysed using 2 theoretical perspectives: conversation analysis and the study of institutional interaction.

\section{Main findings}

A central component of the interaction between informal carers and healthcare workers was the way information and access to information were obtained. The person needing information first had to identify whom to ask and then initiate the request. The recipients of the request then had to decide what information the enquirer would be given. Interactions showed that informal carers treated gate keeping as a legitimate activity of healthcare workers. They did this by cautiously soliciting healthcare workers' help to access other healthcare workers or collections of institutional information, and they avoided undermining the healthcare workers' gate keeping behaviours. This was done by a collaborative use of polite discourse by both informal caregivers and healthcare workers. Negative (or cautious) politeness strategies were particularly important and seemed to be institutionally rather than personally driven; they were used to assume approved social attributes and to avoid being judged negatively (by using qualifiers such as "probably", "perhaps", or "might"). In a few interactions, the informal caregiver adopted the role of gatekeeper. Informal carers were generally uncomfortable with overtly asserting their knowledge and used hedging devices and tentative assertion. Informal carers used the encounters as an opportunity to solicit the opinions of healthcare workers and to access particular expert knowledge. In this way, both parties collaborated in the legitimisation of knowledgeability and competence. However, if the delivery of advice by healthcare workers was not based on a shared footing, they ran the risk of the information being badly received.

\section{Conclusion}

Gate keeping and legitimisation were central elements of the interactions and relationships between informal carers of older people and healthcare workers.

\section{COMMENTARY}

Expert and experienced nurses seek information about patients from a number of sources in order to "know" their patients; information about patient particulars helps nurses to individualise care under various circumstances. ${ }^{12}$ Moreover, patients seek information from nurses and others in order to become more equal partners in care. ${ }^{3}$ The study by May et al alerts us to how information may be exchanged between healthcare workers and informal carers, and encourages insights about these interactions.

The videotaping method provided data on not only what was said, but also the language patterns and non-verbal behaviours of the participants. Analysis revealed that cautiousness, politeness, and deference characterised interactions. Although these findings cannot be generalised, nurses might consider their interactions with carers in public places, attending to how nurses might be perceived as gatekeepers and how nurses' verbal and non-verbal communications may affect what is learned about patients. Sensitivity to the stance of carers as they solicit information may ease the exchange, helping carers become better informed so that they might participate more fully in decision making. Sensitivity to possible carer gate keeping could help nurses learn the "special knowledge" that informal carers have about patients, thereby facilitating the individualisation of interventions.

These findings encourage further examination of informal carer and healthcare worker interactions to uncover possible ethical issues regarding communication, to determine how nurses legitimise the participation of informal carers, and to uncover possible power differences between the 2 groups. Combined methodologies videotaping, participant observation, and interviewing - would shed light on participants' perceptions of how they were treated during interactions, and their perceptions of the outcomes of interactions. Also, categorising interactions by type of healthcare workers (eg, nurses, therapists, and housekeepers) would help specify implications for nursing practice and for ward management.

Laurel E Radwin, RN, CS, PhD Assistant Professor, Department of Adult and Gerontological Nursing College of Nursing and Health Sciences, University of Massachusetts Boston Boston, Massachusetts, USA

1 Radwin LE. Knowing the patient: a process model for individualized interventions. Nurs Res 1995;44:364-70.

2 Tanner CA, Benner P, Chesla C, et al. The phenomenology of knowing the patient. Image J Nurs Sch 1993;25:273-80.

3 Kermode M. Patients' experiences of nursing interventions during hospitalisation with an AIDS-defining illness. Aust J Adv Nurs 1995;12:20-30. 